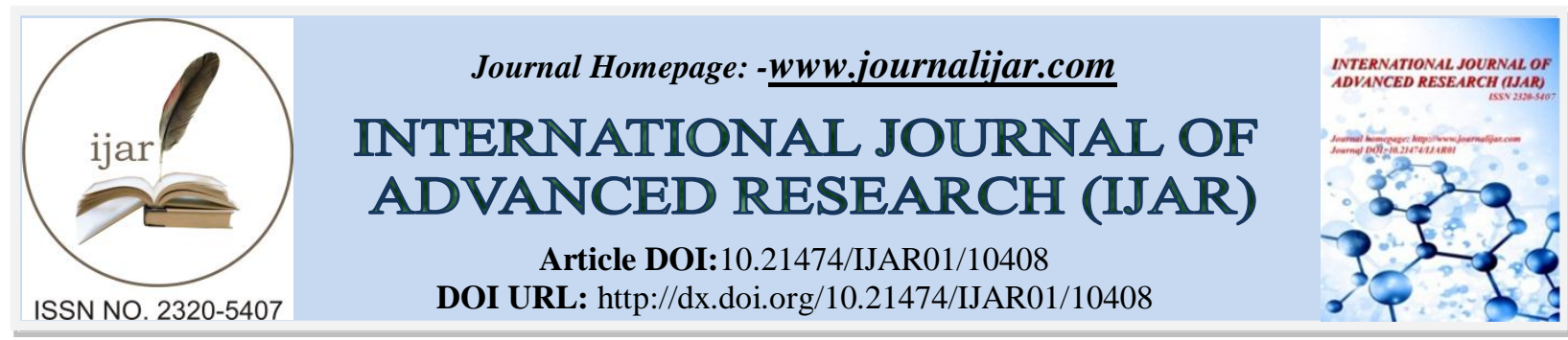

RESEARCH ARTICLE

\title{
DOLOR POSTOPERATORIO ASOCIADO AL USO DE ISQUEMIA EN LOS PACIENTES DE CIRUGÍA ORTOPÉDICA
}

\author{
Esmeralda Hernández Flores ${ }^{1}$, Violeta Dejanira Álvarez Jiménez ${ }^{2}$ and Verónica Herrera Méndez ${ }^{3}$ \\ 1. Calle Manuela Sáenz 293, Colonia Culhuacán CTM Sección VI, C.P. 04480 Coyoacán, CDMX, México. \\ 2. Av. H. Escuela Naval Militar 228, Colonia: Presidentes Ejidales 1ra.Secciòn, C.P. 04470 Coyoacán CDMX, \\ México. \\ 3. Calzada Del Hueso 767, Departamento 202, Colonia: Rinconada Coapa , CDMX, México.
}

\section{Manuscript Info}

………………….

Manuscript History

Received: 30 November 2019

Final Accepted: 31 December 2019

Published: January 2020

Key words:-

Torniquete Neumático, Dolor

Postoperatorio, Cirugía Ortopédica

\section{Abstract}

El torniquete neumático de isquemia se utiliza habitualmente en cirugía ortopédica para permitir el vaciamiento de sangre de las extremidades y proporcionar condiciones óptimas para el desarrollo de la cirugía, sin embargo, con su uso se pueden presentar riesgos asociados tanto a nivel local como a nivel sistémico, una complicación de este es el dolor postoperatorio. Según la ASA, el dolor postoperatorio es el que está presente en el paciente debido a la enfermedad, al procedimiento quirúrgico y a sus complicaciones o a una combinación de ambos, y se caracteriza fundamentalmente por ser un dolor agudo, limitado en el tiempo, predecible y evitable. Su mal control afecta negativamente a la calidad de vida de los pacientes, aumenta el riesgo de complicaciones postquirúrgicas, se asocia a un aumento de la morbilidad y el riesgo de desarrollar dolor crónico persistente. El Objetivo fue evaluar el dolor postoperatorio en los pacientes de cirugía ortopédica con y sin uso de isquemia en el Centro Médico Naval.

Material y Métodos: Se llevo a cabo un estudio de tipo observacional comparativo descriptivo, prospectivo de cohortes para valorar y comparar el dolor postoperatorio, se evaluó un total de 60 pacientes divididos en dos grupos, un grupo con isquemia con una $\mathrm{N}$ de 38 y otro sin uso de isquemia con una $\mathrm{N}$ de 22 pacientes. Se obtuvo la medición de dolor postquirúrgico a las 24 horas de la intervención, mediante la Escala Visual Analógica (EVA).

Resultados: La edad promedio fue 61 a 80 años en ambos grupos, tipo de cirugía la más frecuente fue artroplastia de rodilla en un $68.42 \%$ en el grupo con isquemia y $31.81 \%$ en el grupo sin uso de isquemia, así mismo es la cirugía que presento mayor puntuación de dolor con uso de isquemia presentando dolor moderado en un $50 \%$ y dolor un $18.42 \%$, comparado con el grupo en los que no se usó isquemia presentaron dolor leve 6 de los pacientes en un 27.27\%.Conclusión: El uso de isquemia es una herramienta de gran utilidad en los diversos procedimientos de cirugía ortopédica, sin embargo se han descrito diversas complicaciones con su uso, una de ellas es el dolor postoperatorio el cual es a menudo no valorado de forma adecuada,

Corresponding Author:- Esmeralda Hernández Flores 
cuyá severidad en esta investigación evidenció puntuaciones de dolor altas en la escala de valoración analógica (EVA).

Copy Right, IJAR, 2020,. All rights reserved.

\section{Introduction:-}

El torniquete neumático de isquemia se utiliza habitualmente en cirugía ortopédica para permitir el vaciamiento de sangre de las extremidades, este procedimiento es no invasivo, algunas de sus ventajas de su uso son proporcionar las condiciones óptimas para el desarrollo de la cirugía, así también minimizar el riesgo de sangrado, facilitar la visualización e identificación de las estructuras anatómicas y reducir los tiempos quirúrgicos.

La aplicación del torniquete puede presentar algunas consecuencias fisiológicas y bioquímicas significativas que son relevantes en su manejo transoperatorio y postoperatorio del paciente. Los riesgos asociados al uso de isquemia tienen una serie de inconvenientes tanto a nivel local como a nivel sistémico, siendo una complicación de este el dolor postoperatorio cuya severidad tiene relación directa con el tiempo y la presión a la que se mantiene inflado el torniquete.

El uso de los torniquetes en las extremidades tiene como objetivo evitar pérdidas sanguíneas y mejorar la visión del área quirúrgica, sin embargo, debemos tener en cuenta el potencial daño que puede presentarse después de su uso. La Asociación Internacional para el Estudio del Dolor lo define como una experiencia sensorial y emocional desagradable descrito en términos de daño y asociado a una lesión tisular o potencial. El $90 \%$ de los pacientes presentan dolor postoperatorio, y aproximadamente el $60 \%$ sufre dolor de moderado a intenso en sus primeras 24 horas postquirúrgicas ${ }^{11}$.

La valoración del dolor postoperatorio es una parte esencial de los cuidados perioperatorios ${ }^{3}$. Según la Asociación Americana de Anestesiología (ASA), el dolor postoperatorio es el que está presente en el paciente debido a la enfermedad, al procedimiento quirúrgico y a sus complicaciones o a una combinación de ambos, y se caracteriza fundamentalmente por ser un dolor agudo, limitado en el tiempo, predecible y evitable ${ }^{13}$.

Se han realizado múltiples estudios acerca del uso de isquemia en cirugías de ortopedia, al igual que del dolor postoperatorio.

En un estudio aleatorizado para comparar la penetración de cemento en las radiografías después de la artroplastia total de rodilla (ATR) se realizaron 3 grupos en los cuales el torniquete tenía diferentes usos; el primero fue su aplicación durante la cirugía, el segundo la aplicación solo con prótesis de implantación y el tercero sin uso de torniquete, este estudio concluye que el uso de torniquetes en periodos largos, pueden producir puntuaciones más altas de dolor y reducir la recuperación funcional después de la artroplastia total de rodilla ${ }^{14}$.

En otro estudio Fan Y et al investigaron si el uso limitado del torniquete en artroplastia de rodilla (ATR) reduciría las complicaciones y facilitaría la recuperación postoperatoria, en el cual obtuvieron como resultado que el uso limitado de un torniquete en ATR proporciona una menor inflamación de la extremidad y dolor en la articulación de la rodilla, sin comprometer el tiempo de operación o la pérdida y recuperación de sangre 5 .

Wang et al. Refieren que la aplicación de torniquetes solo durante la cementación podrían reducir complicaciones menores y tener una recuperación funcional rápida durante el período de rehabilitación temprana después de la artroplastia total de rodilla (ATR), pero no podría limitar la pérdida de sangre intraoperatoria y total ${ }^{26}$.

En otro estudio acerca del efecto del uso de torniquetes de larga y corta duración en la artroplastia de rodilla se observó que las pérdidas de sangre totales e intraoperatorias se redujeron con el uso de torniquetes de larga duración, mientras que con el uso de torniquetes de corta duración reducen las pérdidas de sangre postoperatorias y ocultas sin aumentar la tasa de transfusión de sangre alogénica. Además, el uso de torniquetes de corta duración genera una recuperación rápida y menos dolorosa durante el período de rehabilitación temprana después de la ATR 27 .

Zhang et al. Refieren que el uso de un torniquete durante la ATR aumentará el dolor de rodilla y los eventos trombóticos y que es desfavorable para el progreso de la rehabilitación temprana pero que puede disminuir la pérdida total de sangre y la tasa de transfusión ${ }^{29}$. 
$\mathrm{Du} \mathrm{Z}$ et al. Al investigar el efecto del torniquete sobre la pérdida de sangre perioperatoria y la efectividad a corto plazo en la ATR obtuvo como resultado que la aplicación de un torniquete en ATR aumenta la pérdida de sangre oculta, y no existe una ventaja obvia para reducir la tasa de transfusión en comparación con el grupo sin torniquete, por lo que se recomienda reducir el tiempo y la presión del torniquete para pacientes con alto riesgo de trombosis ${ }^{4}$.

Vaishya R et al. El uso de un torniquete de corta duración durante la ATR proporciona un mejor alivio del dolor sintomático en el período postoperatorio temprano en comparación con el uso de un torniquete de larga duración. Sin embargo, esto se asocia con una mayor pérdida de sangre, más tiempo de operación y no tener un campo operatorio claro. Ellos sugieren que se tenga un pensamiento racional y una reconsideración de la práctica del uso rutinario de torniquetes de larga duración en todos y cada uno de los casos de ART $^{24}$.

Liu PL et al. Al evaluar los beneficios del uso de un torniquete en una extremidad en pacientes sometidos a artroplastia total de rodilla (ATR) bilateral simultánea obtuvieron como resultado que el uso de torniquete puede reducir el tiempo intraoperatorio, pero se asoció con una mayor incidencia de complicaciones de la herida y una mayor inflamación postoperatoria de la rodilla ${ }^{10}$.

Azboy L et al. Refieren que después de la ATR, el cierre de la herida con la rodilla en flexión y después de desinflar el torniquete disminuyó significativamente el dolor postoperatorio y promovió la recuperación temprana del rango de movimiento (ROM) en el período postoperatorio temprano ${ }^{20}$.

En el año 2015 en el Instituto Nacional de Ciencias Médicas y Nutrición, se realizó un diagnóstico situacional con respecto al manejo de dolor postoperatorio, en el cual el $63.9 \%$ de los pacientes refirió dolor moderado a severo entre las 6 y 24 horas posteriores al evento quirúrgico, en donde una de las cirugías con mayor incidencia de dolor fue la cirugía ortopédica.

Gómez Morales et al. Al evaluar la atención del dolor postoperatorio concluyeron que el manejo del dolor postoperatorio sigue siendo un problema real y mal controlado, mantiene una prevalencia alta de $48.7 \%$, que se traduce en insatisfacción y sufrimiento de los pacientes ${ }^{7}$.

\section{Historia Del Torniquete:}

Los primeros reportes que se tienen del uso de los torniquetes han sido de Archingenes y Heliodoro contemporáneos de Celso (S.II.d.de.cristo) ellos utilizaban bandas de tela apretadas colocadas en la parte proximal y distal a la zona de incisión, atadas con un solo nudo simple ${ }^{2}$.

Ambrosio Paré (1510-1592) en el siglo XVI. Sugirió la colocación de una goma fuerte y gruesa encima del lugar de la incisión, indicando además que la misma debía realizarse siempre sobre tejido sano. Describió como este tipo de banda ayudaban a controlar la hemorragia, disminuir el dolor y retener la máxima cantidad de piel y músculo para el muñón ${ }^{2}$.

La descripción del sistema sanguíneo por Willian Harver (1578-1657) en 1616, ayudo a desarrollar mejores sistemas de control de sangrado, como las barras enrollada a la goma que permitía aumentar la constricción, cuyo invento se conoció como el "cabestrante español" (Spanish Windiass) ${ }^{2,16}$.

En el siglo XVII se empezó a utilizar el término torniquete, para denominar a estos nuevos sistemas que sirven para limitar la hemorragia. Jean Louis Petil (1674-1750) desarrollo un nuevo modelo que añadía a la banda un tornillo que permitía aumentar la presión debido al funcionamiento del aparato, con giros del tornillo, se le llamo torniquete, del francés “tourner" (girar) ${ }^{2,16}$.

Durante el siglo XIX, se considera que fue Josep Lister (1827-1912), en 1864 fue el primero en emplear la isquemia inducida para intervenciones distintas de amputación, describió las bondades de un campo libre de sangre para la ablación precisa de un tuberculoma en la mano.

Johann Fierdrich August von Esmarch (1823-1908) desarrollo la banda elástica que lleva su nombre, esto permitía evacuar completamente la sangre previamente a la colocación de un torniquete. Actualmente la banda de Esmarch sigue utilizándose para exanguinar el miembro previamente a la cirugía ${ }^{16}$. 
Sin en el siglo $\mathrm{XX}$, todos los sistemas de torniquetes descritos presentaban múltiples complicaciones, como la aparición de daños neurológicos y necrosis muscular por una presión excesiva y la dificultad para colocar y quitar rápidamente si fuese necesario ${ }^{2}$.

Harver Cushing (1869-1939) en 1904 ideo el primer manguito neumático. Modifico los esfingomanotros de Riva Roccio para medir la tensión arterial conectándoles una bomba de bicicleta para facilitar un inflado rápido y adaptando un manómetro para monitorizar la presión ejercida ${ }^{2}$.

\section{Uso Del Torniquete:}

El uso de torniquete es una práctica común en cirugías ortopédicas. El torniquete es un dispositivo de compresión o constricción usado para controlar la circulación hacia una extremidad por un período de tiempo determinado ${ }^{2,6,16}$. En la actualidad, existen diversos tipos de torniquetes: torniquete de emergencia, el cual se coloca alrededor de una extremidad inferior o superior para evitar la pérdida prolongada de sangre por traumatismo y torniquete quirúrgico, el cual permite al cirujano trabajar en un campo quirúrgico sin sangre al evitar el flujo sanguíneo a una extremidad 26

Tienen dos diseños básicos: torniquetes no neumáticos los cuales están hechos de goma o telas elásticas, y torniquetes neumáticos los cuales usan gas comprimido para inflar el manguito y ocluir o restringir el flujo sanguíneo mediante un dispositivo de regulación en la máquina del torniquete que controla la cantidad de presión del manguito ejercida sobre la extremidad ${ }^{2,6,16}$.

La longitud y el ancho del manguito del torniquete deben individualizarse, teniendo en cuenta el tamaño y la circunferencia de la extremidad del paciente. El mango debe ser más ancho que la mitad del diámetro del miembro. Los manguitos más anchos minimizan el riesgo de lesión del tejido subyacente al dispersar la presión sobre un área de superficie mayor. Se ha encontrado que los manguitos más grandes ocluyen sistemáticamente el flujo sanguíneo a una presión más baja en adultos ${ }^{6}$.

La presión a la que se debe inflar un torniquete depende de diversas variables: la edad, piel, presión sanguínea, forma y tamaño de la extremidad del paciente y las dimensiones del manguito. Varios estudios recomiendan presiones del manguito de 200-300 mmHg para adultos. La presión de oclusión en extremidades (POE) se define como la presión mínima requerida para detener el flujo de sangre arterial en la extremidad distal al manguito, se determina aumentando gradualmente la presión del torniquete hasta interrumpir el flujo sanguíneo distal, la POE se ajusta agregando un margen de seguridad de la siguiente manera:

1. Colocar $40 \mathrm{mmHg}$ para $\mathrm{POE}<130 \mathrm{mmHg}$.

2. Colocar $60 \mathrm{mmHg}$ para POE entre $131-190 \mathrm{mmHg}$

3. Colocar $80 \mathrm{mmHg}$ para $\mathrm{POE}>190 \mathrm{mmHg}$,

Se debe evaluar la situación operativa a las dos horas, y si la duración prevista es mayor a 2.5 horas, se debe utilizar un intervalo de deflación de 10 min en ese punto y en los intervalos posteriores de una hora.

Ante el uso frecuente del torniquete en las cirugías ortopédicas se han descrito puntos estratégicos para el uso adecuado de este sistema ${ }^{(30)}$. Bruner estableció 10 normas para el uso seguro del torniquete que han sido modificadas por Braithwaite y Klenerman, las cuales son:

1. Tamaño: $10 \mathrm{~cm}$ para los miembros superiores (MS), $15 \mathrm{~cm}$ para los miembros inferiores (MI) y más ancho en extremidades de mayor tamaño.

2. Lugar de colocación: Parte superior del brazo y medio/superior del muslo.

3. Protección o relleno debajo del manguito: Al menos dos capas de lana(huata) ortopédica.

4. Preparación de la piel: Evitar impregnar el relleno debajo del manguito.

5. Presión: De 50-100 mmHg por encima de la sistólica para los MS y doble de la sistólica para los MI, o de 200 a $250 \mathrm{mmHg}$ para los MS y de 250 a $350 \mathrm{mmHg}$ para los MI.

6. Tiempo: máximo 3 horas (se recupera en 5 a 7 días), pero generalmente no exceder de 2 horas.

7. Temperatura: Evitar el calentamiento, enfriar si es factible y mantener los tejidos húmedos.

8. Monitorización: Controlar la duración y presión.

9. Calibración y mantenimiento al menos semanalmente y el manómetro de mercurio 3 veces mensualmente. 


\section{Complicaciones Del Uso De Torniquete:}

Tiene diferentes efectos entre sistémicos locales y generalizados: síndrome de dificultad respiratoria aguda (SDRA), renal, pueden producirse lesiones cardíacas y pueden dar lugar a otras complicaciones graves y con frecuencia fatales ${ }^{16,23}$.

\section{Las complicaciones relacionadas con el torniquete son:}

1. Lesiones nerviosas, desde parestesia hasta parálisis. Los tejidos nerviosos son menos vulnerables a la lesión aguda en comparación con el musculoesquelético.

2. Lesiones musculares. Se deben al efecto combinado de la isquemia y la deformación mecánica del tejido. La isquemia y la compresión conducen a cambios metabólicos y microvasculares.

\section{Dentro de los efectos sistémicos se encuentran:}

1. Efectos cardiovasculares, los cuales ocurren durante todas las fases de la aplicación del torniquete, desde la exanguinación hasta el desinflado. La exanguinación de las extremidades y la inflación del torniquete aumentan el volumen sanguíneo y la resistencia vascular sistémica que finalmente causan un aumento transitorio de la presión venosa central.

2. Efectos respiratorios principalmente durante el desinflado del torniquete. Se asocia con un aumento transitorio en la tensión del dióxido de carbono al final de la espiración (EtCO2). El aumento en EtCO2 está relacionado con la duración de la isquemia. El aumento de $\mathrm{EtCO} 2$ también es mayor con el torniquete de la extremidad inferior en los hombres que en las mujeres, debido al mayor volumen muscular del hombre ${ }^{16}$.

3. Efectos circulatorios cerebrales posterior a la deflación del torniquete. Aumenta el flujo sanguíneo cerebral en dos minutos y vuelve a la línea de base en 10 minutos ${ }^{9,16}$.

4. Efectos hematológicos con un estado de hipercoagulabilidad secundario al aumento de la agregación plaquetaria y estimulación de los factores de coagulación causados por el daño tisular y catecolaminas liberadas en respuesta al dolor de la cirugía y aplicación del torniquete ${ }^{9,16}$.

5. Cambios metabólicos en la extremidad isquémica que incluyen: aumento de los niveles de ácido láctico, presión parcial de bióxido de carbono $\mathrm{PaCO} 2$.

6. Cambios de temperatura, a nivel central aumenta gradualmente después de la aplicación del torniquete a medida que disminuye el área de superficie disponible para la pérdida de calor, lo que resulta en una menor transferencia de calor desde el compartimiento central al periférico. El desinflado conduce a una caída transitoria en la temperatura central debido a la redistribución del calor corporal y la sangre hipotérmica de la extremidad isquémica ${ }^{9,16}$.

\section{Dolor Postoperatorio:}

Según la Asociación Americana de Anestesiología (ASA), el dolor postoperatorio es el que está presente en el paciente debido a la enfermedad, al procedimiento quirúrgico y a sus complicaciones o a una combinación de ambos, y se caracteriza fundamentalmente por ser un dolor agudo, limitado en el tiempo, predecible y evitable.

El dolor postoperatorio está asociado a un estímulo nocivo, es decir, a un componente de lesión y daño tisular con o sin compromiso visceral que pone en marcha el mecanismo del dolor por activación de los llamados nociceptores. En su producción concurren todos aquellos neuromediadores y neuromoduladores de las vías de conducción y centros integradores del dolor. De alguna manera, estas condiciones ya están establecidas en los pacientes con patologías que requieren tratamiento quirúrgico ${ }^{1,16,18}$.

La intensidad de dolor varia con el acto quirúrgico y cesará con la cicatrización de los tejidos; sin embargo, algunos estados agudos pueden evolucionar a la cronicidad. Cada unidad sensorial incluye un receptor órgano-terminal y una fibra sensorial constituida por un axón acompañante, un ganglio de la raíz dorsal y un axón terminal en la médula espinal. Las vías nerviosas involucradas están previamente normales e intactas. El estímulo generado por daño tisular térmico, mecánico o químico es capaz de activar los nociceptores, que son terminales nerviosas libres (periféricas) ${ }^{23}$.

El término «nocicepción» se deriva de noci (palabra latina que denota daño o lesión) y es usado para describir sólo la respuesta neural a los estímulos traumáticos o nocivos. El dolor nociceptivo se produce por estimulación de los receptores sensitivos específicos o nociceptores localizados con densidad variable en tejidos como la piel, los músculos, las articulaciones y las vísceras. Es precisamente la variación de la densidad de presentación de la población de estos receptores en los tejidos, lo que marca la diferencia sensorial ${ }^{28}$. 
Las fibras nociceptoras son las A- $\delta$ y las $\mathrm{C}$. Las A- $\delta$ son fibras mielínicas de conducción rápida activadas por receptores térmicos, mecanotérmicos y mecanorreceptores de alto umbral. Las fibras C se diferencian de las A- $\delta$ en que son amielínicas, de conducción lenta y con un campo de receptividad menor. Las fibras C presentan la mayoría de los nociceptores periféricos, y la mayoría de ellas son neuronas polimodales, es decir, pueden reaccionar ante estímulos mecánicos, térmicos o químicos ${ }^{12,28}$.

Teniendo en cuenta lo anteriormente expuesto, es posible sintetizar lo que la evidencia ha permitido fundamentar, como propuestas de años recientes, en cuanto a la participación de los nociceptores como pieza clave de la fisiopatología del dolor. Éstas, además de apoyar que la sensación de dolor se produce por la estimulación directa de dichas terminaciones nerviosas, generalmente inducida por la lesión tisular local, también tienen en cuenta el papel que juega en ello la inflamación secundaria al procedimiento quirúrgico, la cual producirá una sensibilización periférica, fenómeno en el que participan sustancias alogénicas, como las prostaglandinas, el potasio, las bradicininas, la histamina, la sustancia $\mathrm{P}$, entre otras ${ }^{16,28}$.

Los nociceptores periféricos son sensibilizados por mediadores tisulares de lesión, aumentan la excitabilidad y la frecuencia de descarga neural. Esta respuesta, también llamada «hiperalgesia primaria», permite que estímulos previamente subnocivos que ingresan a la médula y generen potenciales de acción y sean transducidos ortodrómicamente en la médula espinal. La facilitación de la transducción del impulso en las neuronas de primer orden no sólo es mediada por sustancias nocivas liberadas por los tejidos dañados, también los reflejos axonales exacerban esta reacción al liberar sustancia $P$ neurotransmisor localizado en las vesículas sinápticas de las fibras no mielinizadas C, que causa vasodilatación, desgranulación de mastocitos y, a la vez, esto libera histamina y serotonina y aumenta efectivamente la recepción del campo periférico para incluir tejido adyacente no lesionado 12,28

La generación de los estímulos nocivos es traducida por los nociceptores en impulsos nerviosos y transmitida al sistema nervioso central por las fibras A- $\delta$ y C. Posteriormente, la transmisión de los signos aferentes nociceptivos está determinada por influencias moduladoras complejas en la médula espinal. Allí, en las terminales centrales de las neuronas de primer orden, intervienen los aminoácidos excitatorios L-glutamato, aspartato y varios neuropéptidos, incluyendo el péptido intestinal vasoactivo, la colecistocinina, el péptido liberador de gastrina, la angiotensina II, el péptido relacionado con el gen de la calcitonina y la señalada sustancia $\mathrm{P}^{19}$.

Algunos impulsos nociceptivos pasan a la asta anterior, también a la asta anterolateral para desencadenar respuestas reflejas segmentarias. Otros son transmitidos a los centros superiores a través de los tractos espinotalámicos y espinorreticulares, donde se producen respuestas suprasegmentarias y corticales, que son las que definen la reacción del organismo frente a la agresión y constituyen la base que explica los problemas postquirúrgicos ligados a la presencia del dolor (la «reacción neuroendocrina y metabólica al estrés»). Es así como, mientras la hiperalgesia primaria se desarrolla en la periferia, el fenómeno de «hiperalgesia secundaria» se pone de manifiesto en el sistema nervioso central y precede la sensibilización central. La hiperalgesia secundaria es la fuente para el establecimiento del dolor crónico postoperatorio ${ }^{16,19,28}$.

\section{Escalas De Medición Del Dolor:}

La Escala Visual Analógica (EVA), fue ideada por Scott Huskinson en 1976, es el método de medición empleado con más frecuencia en muchos centros de evaluación del dolor. Consiste en una línea horizontal o vertical de 10 centímetros, que representa la visión continua de la experiencia dolorosa, en cuyos extremos se encuentran las expresiones extremas de un síntoma. En el izquierdo se ubica no dolor y en el derecho el peor dolor imaginable. Su principal ventaja radica en el hecho de que no contienen números o palabras descriptivas, que puedan sugestionar al paciente, sino que es libre de indicarnos sobre la línea continua la intensidad de su sensación dolorosa. La intensidad se expresa en centímetros o milímetros.

\section{Materiales Y Métodos:-}

Se llevo a cabo un estudio de tipo descriptivo, observacional comparativo, prospectivo de cohortes en el Centro Médico Naval para valorar y comparar el dolor postoperatorio asociado al uso de isquemia en los pacientes de cirugía ortopédica. En este tipo de estudio los individuos son identificados en función de la presencia o ausencia de exposición a un determinado factor ${ }^{17}$.

Se asignaron dos grupos diferentes de trabajo a los que se les denomino de la siguiente forma: 
1. Grupo A: Pacientes en los que se usó isquemia durante su procedimiento quirúrgico.

2. Grupo B: Pacientes en los que no se usó isquemia durante su procedimiento quirúrgico.

Los datos demográficos y clínicos se obtuvieron del expediente clínico de los registros de las historias clínicas de los pacientes, así como también de las hojas de enfermería y las hojas de registro de anestesiología.

Los datos de intensidad de dolor postoperatorio se obtuvieron a través de una entrevista y mediante un cuestionario de evaluación de dolor que consta de 10 preguntas, referido por los pacientes y así como de los registros en la hoja de enfermería durante las primeras 24 horas.

De igual forma a través de la Escala Visual Analógica (EVA). La Escala Visual Analógica (EVA) permite medir la intensidad del dolor que describe el paciente con la máxima reproducibilidad entre los observadores. Consiste en una línea horizontal de 10 centímetros, en cuyos extremos se encuentran las expresiones extremas de un síntoma.

Mediante un diseño probabilístico paramétrico. Los datos recolectados fueron procesados usando el programa estadístico Sigma Plot versión 12.0 y el programa SPSS 22 para Windows. Las variables cuantitativas se expresan en medias \pm la desviación estándar y las variables cualitativas son expresadas en números y porcentajes (\%). El comportamiento de las variables fue comparado usando la prueba de normalidad de Kolmogorov-Smirnov. Se usó $t$ de Student para el análisis de variables cuantitativas que siguen una distribución normal, y para variables cualitativas que no siguen una distribución normal se utilizó prueba de Kruskal-Wallis y U de Mann-Whitney. Se consideró significativa una $\mathrm{p}<0.05$.

\section{Resultados:-}

En la presente investigación, se evaluaron un total de 60 pacientes de divididos en dos grupos, un grupo con isquemia con una $\mathrm{N}$ de 38 de los cuales el $36,34 \%$ fueron hombres y el $63.15 \%$ mujeres y otro sin uso de isquemia con una $\mathrm{N}$ de 22 pacientes de los cuales el $36.36 \%$ fueron mujeres y el $63.63 \%$ hombres.

Los resultados del análisis estadístico inferencial muestran que el grupo al cual se indujo isquemia, por medio del uso de torniquete muestran niveles de dolor con diferencia estadísticamente significativa en comparación al grupo que no se aplicó torniquete.

Los resultados indican que el grupo al cual se le indujo isquemia transitoria con uso de torniquete presentó niveles mayores de dolor. Este comportamiento se repitió posterior a las 24 horas en el postoperatorio.

\begin{tabular}{|c|c|c|c|}
\hline 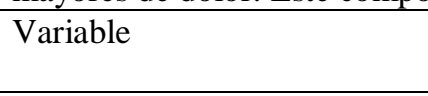 & $\begin{array}{l}\text { Con isquemia } \\
\mathrm{N}=38(63.33 \%)\end{array}$ & $\begin{array}{l}\text { Sin isquemia } \\
\mathrm{N}=22(36.66 \%)\end{array}$ & $\mathrm{P}$ \\
\hline \multicolumn{3}{|l|}{ Edad (promedio y SD) } & \\
\hline 20 a 40 años, n (\%) & $6(15.78)$ & $5(22.72)$ & \multirow{4}{*}{0.756} \\
\hline 41 a 60 años, n (\%) & $12(31.57)$ & $4(18.18)$ & \\
\hline 61 a 80 años, n (\%) & $17(44.73)$ & $10(45.45)$ & \\
\hline Más de 80 años, n (\%) & $3(7.89)$ & $3(13.63)$ & \\
\hline \multicolumn{4}{|l|}{$\operatorname{Sexo}(\mathrm{H}=22, \mathrm{M}=38)$} \\
\hline Hombre, n (\%) & $14(36.84)$ & $8(36.36)$ & \multirow[t]{2}{*}{0.978} \\
\hline Mujer, n (\%) & $24(63.15)$ & $14(63.63)$ & \\
\hline \multicolumn{3}{|l|}{ Tipo de cirugía } & \\
\hline RAFI de radio, $\mathrm{n}(\%)$ & $2(5.26)$ & $3(13.63)$ & \multirow{6}{*}{$<0.001 * * *$} \\
\hline RAFI de cubito, $\mathrm{n}(\%)$ & $3(7.89)$ & $0(0)$ & \\
\hline Artroplastia de rodilla, $\mathrm{n}(\%)$ & $26(68.42)$ & $7(31.81)$ & \\
\hline RAFI de tibia, n (\%) & $0(0)$ & $5(22.72)$ & \\
\hline RAFI de peroné, n (\%) & $0(0)$ & $4(18.18)$ & \\
\hline RAFI de maléolo, n (\%) & $7(18.42)$ & $3(13.63)$ & \\
\hline \multicolumn{4}{|l|}{ Tiempo de cirugía } \\
\hline 60 min o menos, $\mathrm{n}(\%)$ & $3(7.89)$ & $3(13.63)$ & \multirow{4}{*}{0.264} \\
\hline Entre 60 y $90 \mathrm{~min}, \mathrm{n}(\%)$ & $14(36.84)$ & $10(45.45)$ & \\
\hline Entre 90 y $120 \mathrm{~min}, \mathrm{n}(\%)$ & $14(36.84)$ & $9(40.90)$ & \\
\hline Más de 150 min, n (\%) & $7(18.42)$ & $0(0)$ & \\
\hline Frecuencia de dolor en $24 \mathrm{~h}$ & & & \\
\hline
\end{tabular}




\begin{tabular}{|c|c|c|c|}
\hline Ocasionalmente, n (\%) & $9(23.68)$ & $19(86.36)$ & \multirow[t]{3}{*}{$<0.001 * * *$} \\
\hline Frecuentemente, n (\%) & $28(73.68)$ & $3(13.63)$ & \\
\hline Continuo, n (\%) & $1(2.63)$ & $0(0)$ & \\
\hline \multicolumn{3}{|c|}{ Intensidad de dolor en $24 \mathrm{~h}$} & \\
\hline Leve, n (\%) & $0(0)$ & $15(68.18)$ & \multirow{3}{*}{$0.002 * *$} \\
\hline Moderado, n (\%) & $30(78.94)$ & $6(27.27)$ & \\
\hline Severo, n (\%) & $8(21.05)$ & $1(4.54)$ & \\
\hline
\end{tabular}

Tabla 01:- Distribución de grupos con y sin isquemia.

Sin embargo, aunque los grupos se comportaron de manera similar en cuanto edad, sexo y tiempo de cirugía, los grupos habían presentado diferencia estadísticamente significativa en cuanto al tipo de cirugía realizada entre el grupo expuesto a isquemia y el no expuesto, por lo que teorizamos la posible intervención de esta variable como responsable del incremento en la sensación de dolor.

Para verificar si el nivel de dolor estaba determinado por el tipo de cirugía, se realizó un segundo análisis enfocado en cuantificar los niveles de dolor basados exclusivamente en el tipo de cirugía y si es que alguno de estos tipos predispone a incrementar los niveles de dolor. Este análisis se realizó tanto a la muestra completa de pacientes, incluyendo grupos expuestos a isquemia como no expuestos, como en los tipos de cirugía que solo se encontraban en un grupo en particular (expuesto o no expuesto). Los resultados fueron similares en ambos casos siendo que el tipo de cirugía no determinaba un incremento en los niveles de dolor. Los resultados se pueden observar en la siguiente tabla 02 .

\begin{tabular}{|c|c|c|c|c|c|}
\hline Variable & $\begin{array}{l}\text { Artroplastia de } \\
\text { rodilla } \\
\mathrm{N}=33\end{array}$ & $\begin{array}{l}\text { RAFI de radio } \\
\text { y cubito } \\
\mathrm{N}=8\end{array}$ & $\begin{array}{l}\text { RAFI } \\
\text { maléolo } \\
\mathrm{N}=10\end{array}$ & $\begin{array}{l}\text { RAFI de tibia y } \\
\text { peroné } \\
\mathrm{N}=9\end{array}$ & $\mathrm{P}$ \\
\hline \multicolumn{6}{|c|}{$\begin{array}{l}\text { Frecuencia de dolor en } 24 \\
\mathrm{~h}\end{array}$} \\
\hline Ocasionalmente, $\mathrm{n}$ & 12 & 6 & 4 & 6 & \\
\hline Frecuentemente, $\mathrm{n}$ & 20 & 2 & 6 & 3 & 0.134 \\
\hline Continuo, $\mathrm{n}$ & 1 & 0 & 0 & 0 & \\
\hline \multicolumn{6}{|l|}{$\begin{array}{l}\text { Intensidad de dolor } \\
\text { en } 24 \mathrm{~h}\end{array}$} \\
\hline Leve, $n$ & 17 & 2 & 5 & 6 & \\
\hline Moderado, $\mathrm{n}$ & 9 & 6 & 4 & 2 & 0.839 \\
\hline Severo, $\mathrm{n}$ & 7 & 0 & 1 & 1 & \\
\hline
\end{tabular}

Tabla 02:- Distribución del tipo de cirugía.

Teniendo descartada la intervención de la variable "tipo de cirugía" en el incremento de los niveles de dolor, realizamos un siguiente análisis para determinar si las diferencias en la presión ejercida durante el torniquete para inducir isquemia era el responsable de generar un incremento en los niveles de dolor. Para este análisis se subdividió al grupo expuesto a isquemia en 2 subtipos: el primero al cual se indujo isquemia con un torniquete con una presión de entre $200-250 \mathrm{mmHg}$ y el segundo, el cual se indujo isquemia con un torniquete con una presión de entre $250-300 \mathrm{mmHg}$.

Los resultados mostraron que no hubo diferencias estadísticamente significativas en cuanto a los niveles de dolor que presentaron ambos grupos tanto al inicio como $24 \mathrm{~h}$ después y que tampoco hubo diferencia entre necesitar o no más medicación analgésica. Sin embargo, la distribución que siguió este grupo al ser subdividido mostro que este no era un grupo comparable al $100 \%$ puesto que tenían diferencias en variables como la edad, el tipo y el tiempo de cirugía.

Nuestro análisis previo indica la nula participación de estas variables en la génesis del dolor postoperatorio e indica que tanto el tiempo como el tipo de cirugía practicada en estos grupos no modifican la presencia de dolor. De igual manera recomendamos incrementar el número de pacientes para lograr homogeneizar la muestra y que los grupos sean similares en estas variables para poder conocer de mejor manera la intervención de cada variable en la génesis del dolor. Los resultados se observan en la siguiente tabla 03 . 


\begin{tabular}{|c|c|c|c|}
\hline Variable & $\begin{array}{l}\text { Presión } 200-250 \mathrm{mmHg} \\
\mathrm{N}=9\end{array}$ & $\begin{array}{l}\text { Presión } 250-300 \mathrm{mmHg} \\
\mathrm{N}=29\end{array}$ & $\mathrm{P}$ \\
\hline \multicolumn{3}{|l|}{ Edad (promedio y SD) } & \\
\hline 20 a 40 años, n (\%) & $5(55.55)$ & $1(3.44)$ & \multirow{4}{*}{$0.004 * *$} \\
\hline 41 a 60 años, n (\%) & $2(22.22)$ & $10(34.48$ & \\
\hline 61 a 80 años, n (\%) & $2(22.22)$ & $15(51.72)$ & \\
\hline$>80$ años, $\mathrm{n}(\%)$ & $0(0)$ & $3(10.34)$ & \\
\hline \multicolumn{4}{|l|}{$\operatorname{Sexo}(H=22, M=38)$} \\
\hline Hombre, n (\%) & $4(44.44)$ & $10(34.48)$ & \multirow[t]{2}{*}{0.608} \\
\hline Mujer, n (\%) & $5(55.55)$ & $19(65.51)$ & \\
\hline \multicolumn{3}{|l|}{ Tipo de cirugía } & \\
\hline RAFI de radio y cubito, $\mathrm{n}(\%)$ & $3(33.33)$ & $0(0)$ & \multirow[b]{4}{*}{$<0.001 * * *$} \\
\hline Artroplastia de rodilla, n (\%) & $1(11.11)$ & $25(86.2)$ & \\
\hline RAFI de tibia y peroné, n (\%) & $4(44.44)$ & $0(0)$ & \\
\hline RAFI de maléolo, $\mathrm{n}(\%)$ & $3(33.33)$ & $4(13.79)$ & \\
\hline \multicolumn{4}{|l|}{ Tiempo de cirugía } \\
\hline$<60 \min , \mathrm{n}(\%)$ & $3(33.33)$ & $0(0)$ & \multirow{4}{*}{$<0.001 * * *$} \\
\hline Entre 60 y $90 \mathrm{~min}, \mathrm{n}(\%)$ & $5(55.55)$ & $9(31.03)$ & \\
\hline Entre 90 y 120 min, n (\%) & $1(11.11)$ & $20(68.96)$ & \\
\hline$>150 \min , \mathrm{n}(\%)$ & $0(0)$ & $0(0)$ & \\
\hline \multicolumn{4}{|l|}{ Frecuencia de dolor en $24 \mathrm{~h}$} \\
\hline Ocasionalmente, $\mathrm{n}(\%)$ & $3(33.33)$ & $6(20.68)$ & \multirow[t]{3}{*}{0.395} \\
\hline Frecuentemente, $\mathrm{n}(\%)$ & $6(66.66)$ & $22(75.86)$ & \\
\hline Continuo, $\mathrm{n}(\%)$ & $0(0)$ & $1(3.44)$ & \\
\hline \multicolumn{3}{|l|}{ Intensidad de dolor en $24 \mathrm{~h}$} & \\
\hline Leve, n (\%) & $0(0)$ & $13(44.82)$ & \multirow{3}{*}{0.416} \\
\hline Moderado, n (\%) & $9(100)$ & $8(27.58)$ & \\
\hline Severo, n (\%) & $0(0)$ & $8(27.58)$ & \\
\hline
\end{tabular}

Tabla 03:- Distribución de la presión utilizada.

\section{Discusión:-}

En presente estudio tuvo como objetivo general, evaluar el dolor postoperatorio en los pacientes de cirugía ortopédica con y sin uso de isquemia del Centro Médico Naval, en nuestra población se evaluaron un total de 60 pacientes encontrando una mayor prevalencia de mujeres. Se obtuvo como resultado que en la población de estudio todos experimentaron dolor postoperatorio con variaciones en su intensidad de acuerdo a la escala de valoración analógica (EVA), lo que concuerda con la literatura acerca de que la prevalencia del dolor postoperatorio no ha disminuido a pesar del mayor conocimiento de su manejo, debido a que no existe ninguna cirugía donde no duela absolutamente nada ${ }^{(34)}$,resultados que al ser comparados con lo encontrado por parte del el Instituto Nacional de Ciencias Médicas y Nutrición al realizar un diagnóstico situacional con respecto al manejo de dolor postoperatorio, en el cual el $63.9 \%$ de los pacientes refirió dolor moderado a severo entre las 6 y 24 horas posteriores al evento quirúrgico, en donde una de las cirugías con mayor incidencia de dolor fue la cirugía ortopédica. Por otro lado, Gómez Morales et al. Al evaluar la atención del dolor postoperatorio concluyeron que el manejo del dolor postoperatorio sigue siendo un problema real y mal controlado, mantiene una prevalencia alta de $48.7 \%$, que se traduce en insatisfacción y sufrimiento de los pacientes ${ }^{7}$.

Al comparar la frecuencia del dolor postoperatorio en los pacientes con y sin isquemia se obtuvo que los pacientes en los cuales se usa isquemia en un 73.68\% presentaron frecuentemente dolor en las primeras 24 horas, lo cual tiene relación directa con el uso del torniquete, datos que al ser comparados con lo encontrado por la autora Patricia et al. A cerca del dolor postoperatorio: frecuencia y caracterización del manejo al determinar la frecuencia del dolor en los pacientes postoperados obtuvo como resultado que la frecuencia es dolor moderado, severo o insoportable en un $66.3 \%$. 
Los resultados obtenidos al comparar la intensidad de dolor en los dos grupos de estudio con uso y sin uso de isquemia en relación con el tiempo de cirugía (tabla 02), datos que la ser comparados con lo encontrado por Montoya et al. El dolor severo en el postoperatorio es uno de los factores de riesgo más consistentes, para el desarrollo de dolor crónico postquirúrgico, se presenta del 10\% hasta el 50\% de los pacientes después de la cirugía

${ }^{13}$. Por otro lado, Jiang FZ et al. Afirman que al comparar puntuación del dolor en el momento de la aplicación del torniquete fue mayor en el grupo de torniquete de anillo de silicona (SRT), pero la alteración en las puntuaciones de dolor en el grupo con torniquete neumático $(\mathrm{PT})$ fue más alto, con significación estadística $(\mathrm{P}<0.05)(2)$. Sin embargo, a pesar su uso generalizado, hay complicaciones asociadas al uso de torniquete como explica Tie K et al. Los efectos secundarios no deseados del torniquete ocurren a partir de la isquemia y la reperfusión le lesión en la liberación del torniquete. Estos consisten en lesión muscular, rigidez postoperatoria, dolor, inflamación de las extremidades, parálisis nerviosa, trombosis venosa profunda (TVP), lesión vascular ${ }^{22}$.

No se encontró relación entre el tiempo de cirugía e intensidad de dolor, así como también en la presión utilizada y la intensidad de dolor con y sin el uso de isquemia, no hay diferencia estadística significativa, sin embargo se observó que hay puntuaciones de dolor alto en la escala analógica visual con el uso de isquemia relacionado con los que no se usa isquemia, comparado con los datos obtenidos en el estudio realizado por Ozkunt O et al. En el cual concluyen que no sugieren el uso de torniquetes de larga duración, debido a que puede generar puntuaciones de dolor más altas y reducir la recuperación funcional después de una artroplastia total de rodilla ${ }^{20}$. Por otro lado, hablando del dolor en el posoperatorio inmediato comparando el uso de isquemia y el no uso de isquemia, medido con la Escala Visual Análoga para el dolor se observa que al colocar el sistema de isquemia no presenta una diferencia significativa, obteniendo resultados de 6.20 puntos como media para los pacientes en los cuales se utilizó el sistema de isquemia contra 4.92 en aquellos pacientes en los cuales no se utilizó, sin encontrar una significancia estadística $^{21}$.

Zhang $Q$ et al. explican que la respuesta isquémica es heterogénea entre los individuos después de 45 minutos de isquemia. Quince minutos con una presión de torniquete de $350 \mathrm{~mm} \mathrm{Hg}$ parece ser seguro en pacientes sin otra comorbilidad. No hubo diferencias significativas en el tiempo de operación $(\mathrm{t}=-1.353, \mathrm{P}=0.178)(38)$. Con un control adecuado de la cantidad de presión (menos de $293 \mathrm{~mm} \mathrm{Hg}$ ) y la duración de la aplicación (menos de 150 minutos), la liberación del torniquete después del cierre de la herida puede reducir la pérdida de sangre en pacientes tratados con ATR primaria sin aumentar el riesgo de complicaciones ${ }^{29}$.

\section{Referencias Bibliográficas:-}

1. Badía, W. (2018). Tema I. Fisiopatología del Acto Quirúrgico y Postoperatorio. ARS MEDICA Revista de Ciencias Médicas, 6(18), 4-40.

2. Drosos GI, Kiziridis G, Aggelopoulou C, Galiatsatos D, Anastassopoulos G, Ververidis A, Kazakos K.Arch Bone Jt Surg. 2016 enero; 4 (1): 16-22.

3. Esteve-Pérez N, Sansaloni-Perelló C, Verd-Rodríguez M, Ribera-Leclerc H, Mora-Fernández C. Nuevos enfoques en el tratamiento del dolor agudo postoperatorio \% J Revista de la Sociedad Española del Dolor. 2017;24:132-9.

4. Du Z, Liu P, Zhang Y, Li D, Li M. [Effect of tourniquet on perioperative blood loss and short-term effectiveness in total knee arthroplasty]. Zhongguo xiu fu chong jian wai ke za zhi = Zhongguo xiufu chongjian waike zazhi $=$ Chinese journal of reparative and reconstructive surgery. 2013;27(11):1318-23

5. Fan Y, Jin J, Sun Z, Li W, Lin J, Weng X, et al. The limited use of a tourniquet during total knee arthroplasty: a randomized controlled trial. The Knee. 2014;21(6):1263-8.

6. Fitzgibbons, PG, DiGiovanni, C., Hares, S., y Akelman, E. (2012). Uso seguro del torniquete: una revisión de la evidencia. Diario de la Academia Americana de Cirujanos Ortopédicos, 20 (5), 310-319. doi: 10.5435 / jaaos-20-05-310.

7. Gómez Morales CE, García Posada LD, López Garcés VM. Evaluación de atención del dolor postoperatorio en la Unidad Médica de Alta especialidad. León Guanajuato \%J Anestesia en México. 2016;28:20-7.

8. Hicks RW, Denholm B: Implementando las prácticas recomendadas por AORN para el cuidado de pacientes que se someten a procedimientos asistidos por torniquetes neumáticos . AORN J. 2013, 98: 394-396. 10.1016 / j.aorn.2013.08.004

9. Kruse, H., Christensen, KP, Møller, AM, y Gögenur, I. (2015). El uso de torniquetes durante la cirugía de tobillo conduce a un mayor uso de opioides postoperatorios. Diario de anestesia clínica, 27 (5), 380-384. Doi: 10.1016 / j.jclinane.2015.03.034 
10. Liu PL, Li DQ, Zhang YK, Lu QS, Ma L, Bao XZ, et al. Effects of Unilateral Tourniquet Used in Patients Undergoing Simultaneous Bilateral Total Knee Arthroplasty. Orthopaedic surgery. 2017;9(2):180-5.

11. Mouraux, A., Plaghki, L., \& Le Bars, D. (2018). Fisiología del dolor: mecanismos centrales y controles. EMCAnestesia-Reanimación, 44(4), 1-22.

12. Moreno-Monsiváis MG, Fonseca-Niño EE, Interial-Guzmán MG. Factores asociados con la intensidad del dolor en pacientes mexicanos hospitalizados en periodo postoperatorio \% J Revista de la Sociedad Española del Dolor. 2017;24:4-10.

13. Montoya, B. E., Serna, I. D., Guzmán, D. L., López, J. J., Mejía, L. A., \& Restrepo, V. E. (2019). Estudio observacional descriptivo sobre recuperación funcional temprana y dolor postoperatorio en cirugía de reemplazo articular de rodilla.Revista Española de Cirugía Ortopédica y Traumatología, 63(6), 389-393.

14. Ozkunt O, Sariyilmaz K, Gemalmaz HC, Dikici F. The effect of tourniquet usage on cement penetration in total knee arthroplasty: A prospective randomized study of 3 methods. Medicine. 2018;97(4):e9668.

15. Pérez-Guerrero AC, Aragón MC, Torres LM. Dolor postoperatorio: ¿hacia dónde vamos? \%J Revista de la Sociedad Española del Dolor. 2017;24:1-3.

16. Plaghki, L., Mouraux, A., y Le Bars, D. (2018). Fisiología del dolor. EMC - Kinesiterapia - Medicina Física, 39 (1), 1-22. doi: 10.1016 / s1293-2965 (18) 88603-0

17. Pita Fernández, S. Epidemiología. Conceptos básicos. En: Tratado de Epidemiología Clínica. Madrid; DuPont Pharma, S.A.; Unidad de epidemiología Clínica, Departamento de Medicina y Psiquiatría. Universidad de Alicante: 1995. p. 25-47.

18. Ramos-Chávez, V. M. (2018). Síndrome de reperfusión con el uso de torniquete neumático. Revista Mexicana de Anestesiología, 41(S1), 139-143.

19. Ramos-Alanis, A., Guajardo-Rosas, J., Chejne-Gómez, F., Juáres-Lemus, A. M., \& Ayón-Villanueva, H. (2018). Mecanismos para prevenir dolor agudo a crónico. Rev Mex Anest, 41(Supl 1).

20. Sukur E, Ozturkmen Y, Akman YE, Senel A, Azboy I. The effect of tourniquet and knee position during wound closure after total knee arthroplasty on early recovery of range of motion: a prospective, randomized study. Archives of orthopaedic and trauma surgery. 2016;136(12):1773-80.

21. Use of a tourniquet in total knee arthroplasty: a systematic review and meta-analysis of randomized controlled trials.Jiang FZ, Zhong HM, Hong YC, Zhao GF.J Orthop Sci. 2015 Jan; 20(1):110-23. Epub 2014 Nov 6

22. Tie K, Hu D, Qi Y, Wang H, Chen L.Orthopedics. Effects of Tourniquet Release on Total Knee Arthroplasty.2016 Jul 1; 39(4):e642-50. Epub 2016 Jun 13. https://doi.org/10.3928/01477447-20160606-03

23. Valle, A. F., \& AgullaIV, T. A. (2018). El dolor agudo posoperatorio, aún un problema en el paciente quirúrgico. Revista Cubana de Pediatría, 90(3).

24. Vaishya R, Agarwal AK, Vijay V, Tiwari MK. Short term outcomes of long duration versus short duration tourniquet in primary total knee arthroplasty: A randomized controlled trial. Journal of clinical orthopaedics and trauma. 2018;9(1):46-50.

25. Wakai A, Winter DC, Street JT, Redmond PH: Torniquetes neumáticos en cirugía de extremidades . J Am Acad Orthop. 2001, 9: 345-51.

26. Wang C, Zhou C, Qu H, Yan S, Pan Z. Comparison of tourniquet application only during cementation and long-duration tourniquet application in total knee arthroplasty: a meta-analysis. Journal of orthopaedic surgery and research. 2018;13(1):216

27. Wang K, Ni S, Li Z, Zhong Q, Li R, Li H, et al. The effects of tourniquet use in total knee arthroplasty: a randomized, controlled trial. Knee surgery, sports traumatology, arthroscopy: official journal of the ESSKA. 2017;25(9):2849-57

28. Zuñiga, S. T., \& Tarraza, G. B. (2018). Medición del dolor. ARS MEDICA Revista de Ciencias Médicas, 23(3).

29. Zhang Q, Dong J, Gong K, Li X, Zheng S, Wen T.Zhongguo Xiu Fu Chong Jian Wai Ke Za Zhi. Efectos del uso del tourniquet en el resultado perioperatorio en arrotastia total de la rodilla. 2016 Apr; 30(4):421-5. 